\title{
Vertical vibrations of circular flexible foundations on layered media
}

\author{
Nenad Gucunski \\ Department of Civil and Environmental Engineering, Rutgers University, Piscataway, New Jersey 08855-0909, USA \\ $\boldsymbol{\&}$ \\ Ralf Peek \\ Department of Civil and Environmental Engineering, The University of Michigan, Ann Arbor, Michigan 48109, USA
}

(Received 12 January 1993; revised version received 29 March 1993; accepted 31 March 1993)

\begin{abstract}
The paper presents the analysis of vertical oscillations of a flexible circular plate on the surface of an elastic half-space and an elastic layered system by the 'ring method'. The matrix of influence coefficients for the layered system is evaluated based on the stiffness matrix approach, and numerical integration is used to perform the inverse Hankel transforms. The plate discretization is achieved by the finite difference energy method. Vertical oscillations have been analyzed to determine the displacement and soil reaction distributions at the soil-plate interface, and the impedance functions. The results indicate that the response of a flexible plate strongly depends on the material and geometrical properties of both a soil system and a plate, and the load distribution on the plate. In most cases the behavior of a flexible plate differs significantly from the behavior of a rigid one.
\end{abstract}

\section{INTRODUCTION}

Vertical oscillations of rigid, massless, circular foundations at the surface of an elastic half-space have been investigated for more than 50 years. Richart et al. ${ }^{1}$ and Gazetas $^{2}$ summarized earlier solutions. The problem of vertical oscillations of rigid and flexible foundations on layered media has been investigated during the last 20 years. Studies by Luco, ${ }^{3,4}$ Kausel, $^{5}$ Tassoulas ${ }^{6}$ and Wolf and Darbre 7 deal with rigid disks on layered media, and indicate the strong influence of soil layering on the response of foundations. Some of the studies on the response of flexible foundations are by Lin, ${ }^{8}$ Iguchi and Luco, ${ }^{9,10}$ Riggs and Waas, ${ }^{11}$ Karabalis and Beskos, ${ }^{12}$ Gaitanaros and Karabalis ${ }^{13}$ and Swaddiwudhipong et al. ${ }^{14}$ These studies involved flexible foundations on the surface of an elastic half-space, except the studies by Riggs and Waas, and Swaddiwudhipong et al. which involved the flexible circular and rectangular foundations on a layered stratum on a rigid bedrock, respectively; and the study by Iguchi and Luco ${ }^{10}$ which involved a massless circular plate with a rigid core on a

Soil Dynamics and Earthquake Engineering 0267-7261/93/\$06.00 (C) 1993 Elsevier Science Publishers Ltd. layered viscoelastic half-space. Their results indicate a significant influence of the flexibility of the foundation in its response to dynamic loadings, especially for higher frequency loadings.

In most cases the foundation of a dynamically loaded structure can be described as a flexible plate resting on or embedded into a layered half-space. Therefore the primary objective of this paper is to present the solution to the problem of oscillations of a flexible circular disk at the surface of a layered elastic half-space. The secondary objective is to examine the geometrical and material properties of the plate and the medium for which the behavior significantly differs from that of a rigid plate.

\section{METHOD OF ANALYSIS}

The critical point in the solution of problems of oscillations of plates on an elastic medium constitutes a knowledge of the soil reaction distribution. Many of the earlier solutions of rigid plates are based on the assumption of the soil reaction distribution. ${ }^{1}$ Once the distribution is known, the problem can be treated as any other problem of oscillations of flexible plates. The 
Table 1. The stiffness matrix of a layer in cylindrical coordinates ${ }^{21}$

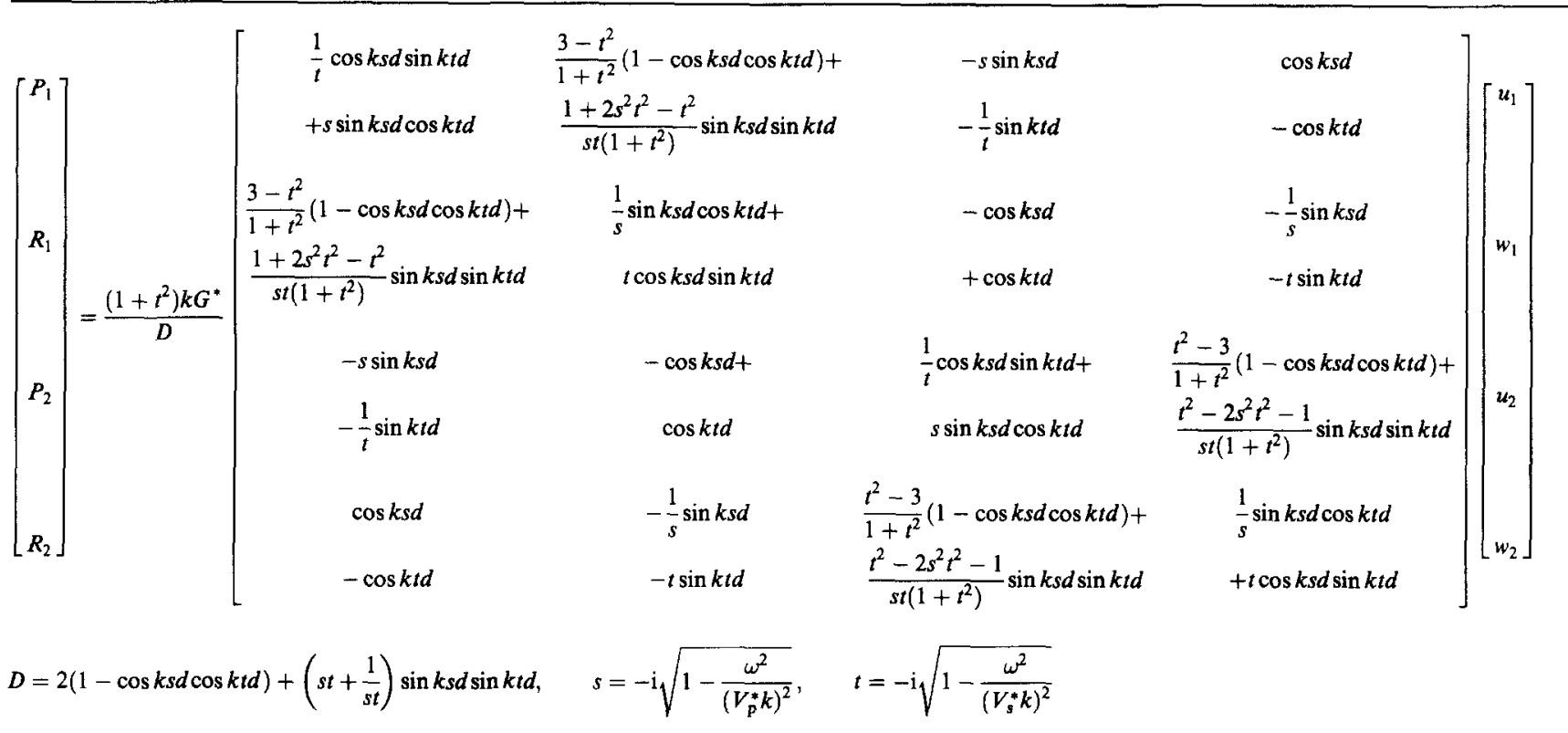

procedure used for the analytical solution of this problem is based on the 'ring method'. 15,16 The soil reaction is assumed to be constant within each of a set of rings. The pressure in each ring is then determined from a compatibility of displacements. In the case of a rigid footing the displacements for all rings must be equal. In the case of a flexible plate the compatibility requires the vertical displacement of the plate to be equal to that of the soil. Thus a lubricated disk-soil interface is assumed.

To define the relationship between the soil reaction and displacement distributions, the stiffness matrix approach was used. The part of the solution representing the response of the layered system is based on the solution for the stiffness matrix of a layer in the frequency-wave number domain. ${ }^{17}$ The part of the solution representing the response of the plate is based on the finite difference energy method (FDEM) in the derivation of the stiffness matrix of a circular plate. The following sections describe (1) the stiffness-matrix approach in problems of soil-structure interaction, (2) the FDEM approach in the derivation of the stiffness matrix of the circular plate, and (3) combination of these two elements in the solution of vertical oscillations of a flexible plate.

\section{The stifiness-matrix approach in problems of soil-structure interaction}

The stiffness-matrix approach corresponds to the stiffness or the displacement method in structural analysis. The stiffness matrix for the layered soil medium is assembled from the stiffness matrices of the soil layers and the half-space in the same way as the stiffness matrix of a structure is assembled from the stiffness matrices of structural elements. The major difference is that the stiffness matrices of layers and the half-space are evaluated in the domain of frequency-wave number instead of the common spatial domain. The equilibrium equation of the soil system is presented by

$$
\mathbf{S}(k) \mathbf{u}(k)=\mathbf{q}(k)
$$

where $\mathbf{S}$ is the stiffness matrix of the soil system, $\mathbf{u}$ the vector of layer interface displacements, $q$ the vector of external layer interface loadings, and $\mathbf{k}$ the wave number. Wave number $\mathbf{k}$ is defined as the ratio $\omega / c$, where $\omega$ is circular frequency and $c$ the phase velocity.

The stiffness matrix of a layer was presented by Kausel and Roesset ${ }^{17}$ as an extension of the transfer matrix formulation by Thomson ${ }^{18}$ and Haskell. ${ }^{19}$ The stiffness matrix was later represented in a slightly modified form by Wolf and Obernhuber ${ }^{20}$ and Wolf. ${ }^{21}$ The stiffness matrix of a half-space can be derived from the stiffness matrix of a layer by implementing the radiation condition, i.e. no incoming waves present in the system. Therefore the stiffness-matrix approach efficiently resolves problems of energy radiation in a layered half-space. Since the problem of vertical oscillations of a circular disk is axisymmetric, the stiffness matrices presented in Tables 1 and 2 contain only the in-plane displacement components $u$ (radial)

Table 2. The stifiness matrix of the half-space in cylindrical coordinates $^{21}$

$$
\left[\begin{array}{l}
P_{\mathrm{o}} \\
R_{\mathrm{o}}
\end{array}\right]=k G^{*}\left[\begin{array}{cc}
\frac{\mathrm{i} s\left(1+t^{2}\right)}{1+s t} & 2-\frac{1+t^{2}}{1+s t} \\
2-\frac{1+t^{2}}{1+s t} & \frac{\mathrm{i} t\left(1+t^{2}\right)}{1+s t}
\end{array}\right]\left[\begin{array}{l}
u_{\mathrm{o}} \\
w_{\mathrm{o}}
\end{array}\right]
$$




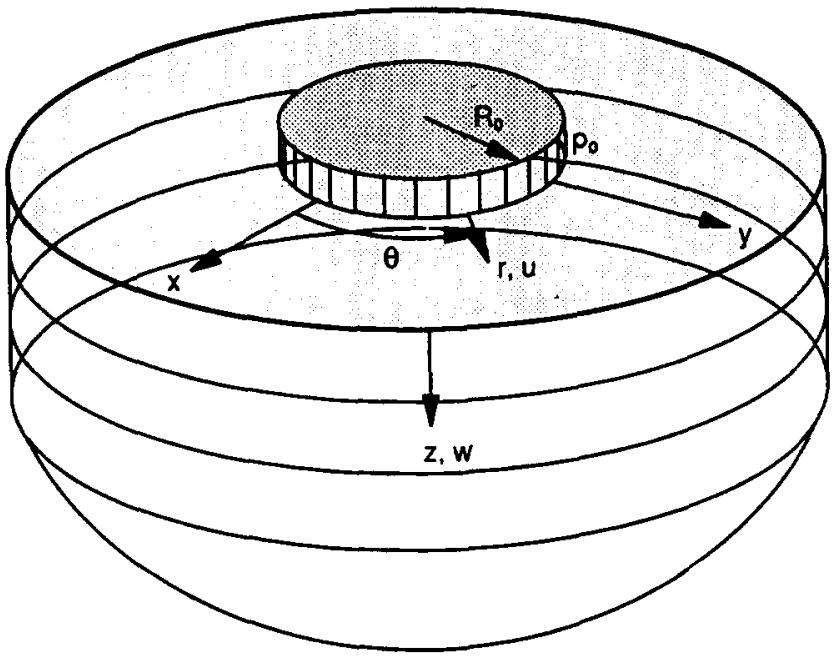

Fig. 1. An axisymmetric model of a soil system with circular loading. ${ }^{22}$

and $w$ (vertical), and loading components $P$ (radial) and $R$ (vertical) (Fig. 1). Modified stiffness matrices for cases when $k$ and $\omega$ take values zero or infinity can be found in Kausel and Roesset ${ }^{17}$ and Wolf ${ }^{21}$ where $G^{*}, V_{p}^{*}$ and $V_{s}^{*}$ are complex shear moduli, $p$-wave and $s$-wave velocities, respectively, and $d$ the thickenss of the layer.

An arbitrary time forcing function can be expressed in terms of its harmonics by using Fourier transforms. Therefore, the following discussion will be concentrated on the frequency domain analysis only. The loading needs first to be transformed from a spatial domain to a frequency-wave number domain. In the case of axisymmetric loading, a common procedure is to expand the loading function in a Fourier series in the circumferential direction $\theta$ and into Bessel functions by Hankel's transforms, involving the frequency wave number $k$, in the radial direction $r$. For a uniform vertical loading $p_{\mathrm{o}}$ acting over a radius $R_{\mathrm{o}}$, the corresponding loading in the wave number domain is

$$
q_{\mathrm{o}}(k)=-\frac{p_{\mathrm{o}} R_{\mathrm{o}}}{k} J_{1}\left(k R_{\mathrm{o}}\right)
$$

where $J_{1}$ is the Bessel function of the first kind of order 0 .

Displacements $u(k)$ and $w(k)$ can be easily obtained by solving eqn (1), where $q_{\mathrm{o}}$ is the only nonzero component of the loading vector $q$. Figure 2 is an example of vertical surface displacements in the frequency wave number domain due to uniform vertical circular loading at the surface of a two-layer over a half-space system. Other values included in the figure represent the layer thickness $d_{\mathrm{j}}$, the Poisson's ratio $\nu$ and the damping ratio $\xi$ of soil. The strong peak displacements represent Rayleigh waves.

To obtain displacements in the spatial domain, the inverse Hankel's transform needs to be applied on the displacements in the wave number domain as the

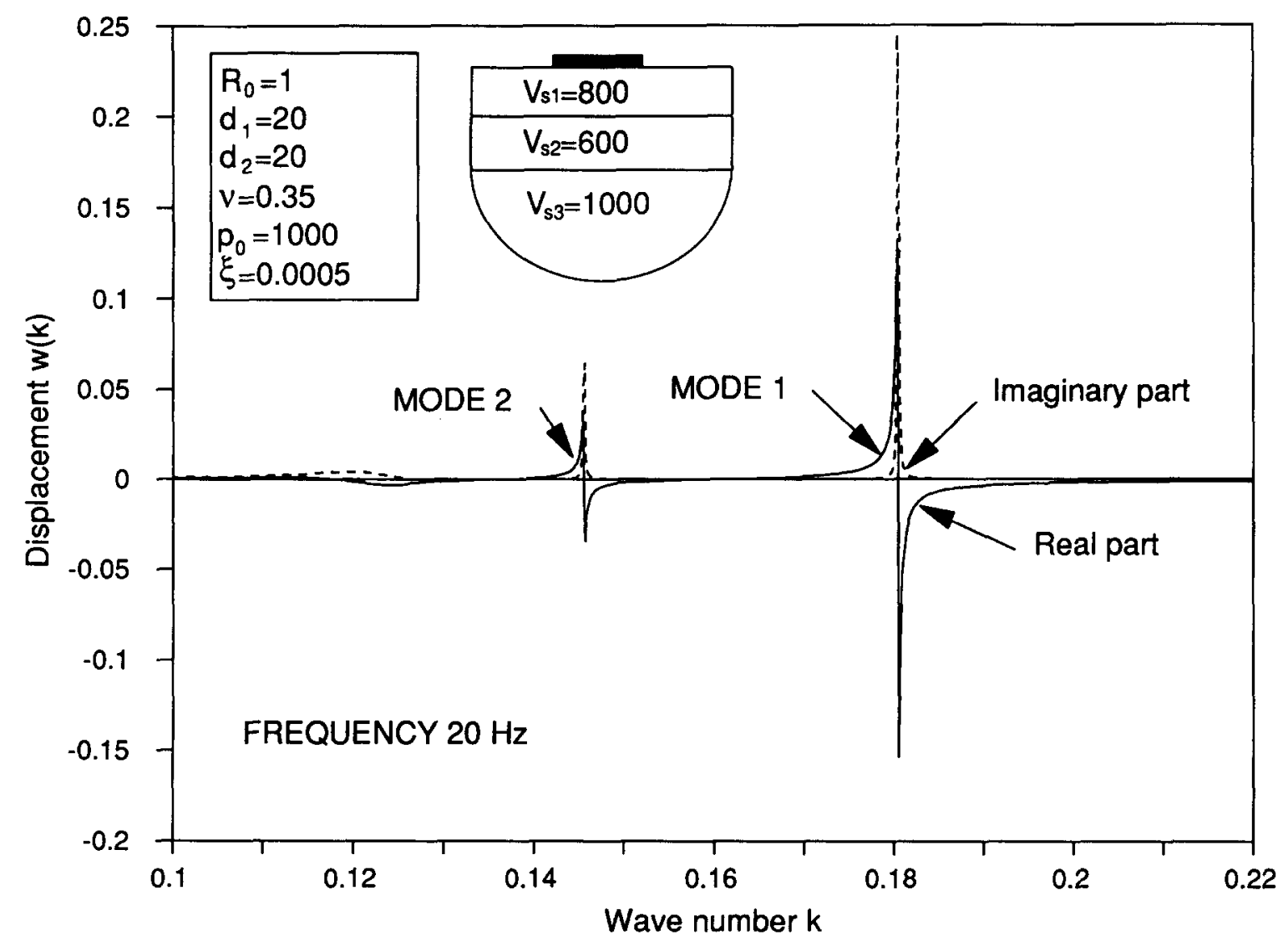

Fig. 2. Vertical displacements in the wave number domain. 


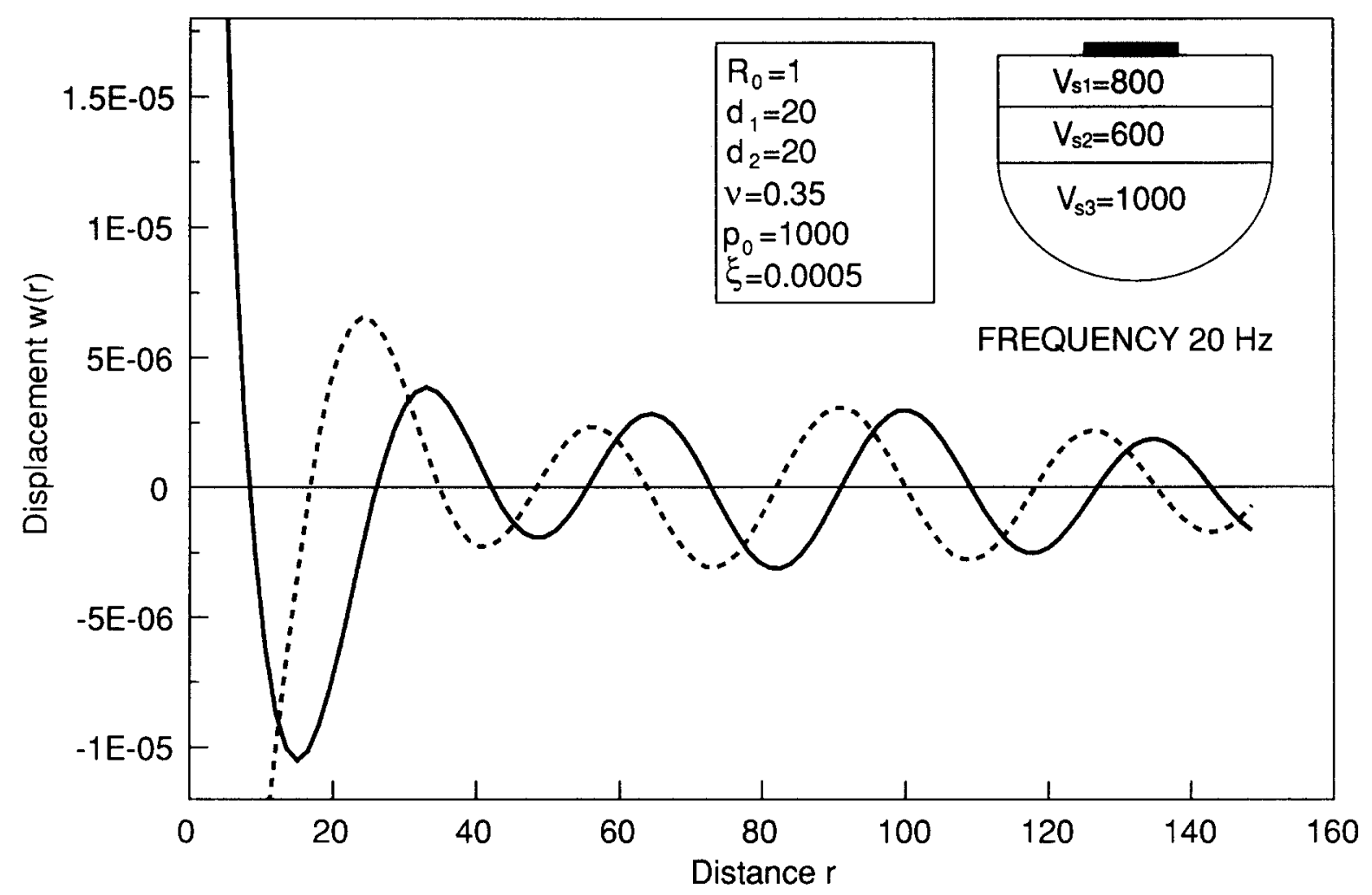

Fig. 3. Surface vertical displacements in the spatial domain. Real part $(-)$, imaginary part $(---)$.

summation in the circumferential direction. General expressions for this operation can be found elsewhere. For this particular problem the surface vertical displacement $w_{\mathrm{so}}(r)$ can be written as

$$
w_{\mathrm{so}}(r)=-p_{\mathrm{o}} R_{\mathrm{o}} \int_{k=0}^{\infty} J_{1}\left(k R_{\mathrm{o}}\right) J_{\mathrm{o}}(k r) w_{\mathrm{o}}^{1}(k) \mathrm{d} k
$$

where $w_{0}^{1}$ represents the surface vertical displacement due to a unit surface vertical loading in the wave number domain. The displacements in Fig. 2 have been numerically integrated according to eqn (3) and the vertical displacements presented in Fig. 3. The integration was performed along the real $k$ axis. A very low material damping was introduced to avoid singularities along the axis for the undamped system.

\section{The FDEM solution for the stiffness matrix of a circular plate}

The solution by the FDEM involves evaluation of the stiffness matrix of the plate through evaluation of the internal virtual work of the plate element. It has been described and successfully implemented by Bushnell. ${ }^{23}$ The difference between the FDEM and the Finite Element Method (FEM) is that in the FDEM displacements are specified at certain nodal points without specifying exactly how the displacements vary between nodal points. Figure 4 shows the discretized model of the plate and corresponding degrees of freedom.

The stiffness matrix of ring $j$ relates displacements and loadings at degrees $j+1, j$ and $j-1$. It is equal to $\mathbf{S}_{p j}=D_{j}$

$$
\begin{aligned}
& {\left[\begin{array}{ccc}
\frac{1}{(\Delta r)^{4}}-\frac{\nu}{r_{j}(\Delta r)^{3}}+\frac{1}{4 r_{j}^{2}(\Delta r)^{2}} & -\frac{2}{(\Delta r)^{4}}+\frac{\nu}{r_{j}(\Delta r)^{3}} & \frac{1}{(\Delta r)^{4}}-\frac{1}{4 r_{j}^{2}(\Delta r)^{2}} \\
-\frac{2}{(\Delta r)^{4}}+\frac{\nu}{r_{j}(\Delta r)^{3}} & \frac{4}{(\Delta r)^{4}} & -\frac{2}{(\Delta r)^{4}}-\frac{\nu}{r_{j}(\Delta r)^{3}} \\
\frac{1}{(\Delta r)^{4}}-\frac{1}{4 r_{j}^{2}(\Delta r)^{2}} & -\frac{2}{(\Delta r)^{4}}-\frac{\nu}{r_{j}(\Delta r)^{3}} & \frac{1}{(\Delta r)^{4}}+\frac{\nu}{r_{j}(\Delta r)^{3}}+\frac{1}{4 r_{j}^{2}(\Delta r)^{2}}
\end{array}\right]} \\
& \times 2 \pi r_{j} \Delta r
\end{aligned}
$$

where $D_{j}$ represents the flexural rigidity of ring $j$ given by

$$
D_{j}=\frac{E_{\mathrm{p}} h_{j}^{3}}{12\left(1-\nu_{\mathrm{p}}^{2}\right)}
$$

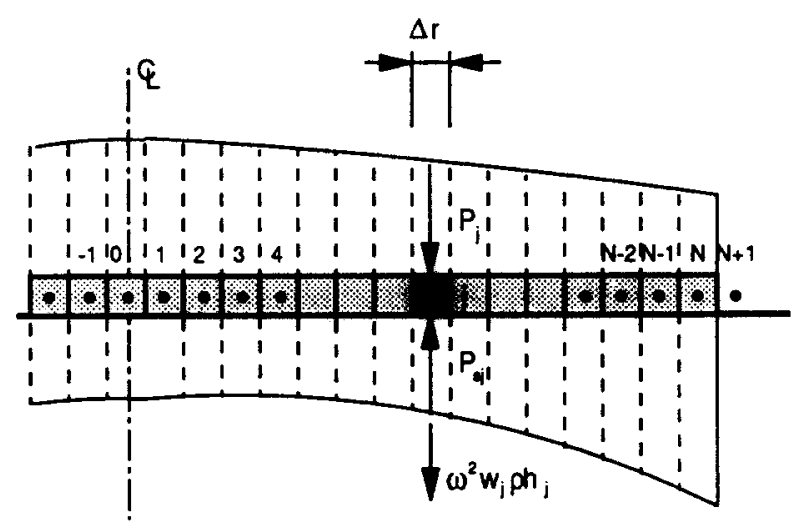

Fig. 4. A schematic of the discretized model of the plate for FDEM. 
$E_{\mathrm{p}}, \nu_{\mathrm{p}}$ and $h$ are the modulus of elasticity, Poisson's ratio and the thickness of the plate, respectively. The loading components in this case are represented by equivalent loadings for the entire ring.

Degrees of freedom $w_{-1}$ and $w_{N+1}$ are eliminated by imposing boundary conditions at the center and at the edge of the plate. This results in special $2 \times 2$ stiffness matrices $\mathbf{S}_{\mathrm{p} 0}$ and $\mathbf{S}_{\mathrm{p} N}$ for rings 0 and $N$. They are equal to

$$
\mathbf{S}_{\mathrm{po}}=\frac{2 D_{\mathrm{o}}}{(\Delta r)^{2}}(1+\nu) \pi\left[\begin{array}{cc}
1 & -1 \\
-1 & 1
\end{array}\right]
$$

and

$\mathbf{S}_{\mathrm{p} N}=$

$$
\left[\begin{array}{cc}
\frac{\left(1+T_{2}\right)^{2}}{(\Delta r)^{4}}+\frac{\nu\left(T_{2}-1\right)\left(1+T_{2}\right)}{R(\Delta r)^{3}} & \frac{\left(1+T_{2}\right)\left(T_{1}-2\right)}{(\Delta r)^{4}}+\frac{T_{1}\left(T_{2}-1\right)}{4 R^{2}(\Delta r)^{2}} \\
+\frac{\left(T_{2}-1\right)^{2}}{4 R^{2}(\Delta r)^{2}} & \frac{\left[\left(1+T_{2}\right) T_{1}+\left(T_{2}-1\right)\left(T_{1}-2\right)\right] \nu}{2 R(\Delta r)^{3}} \\
\frac{\left(1+T_{2}\right)\left(T_{1}-2\right)}{(\Delta r)^{4}}+\frac{T_{1}\left(T_{2}-1\right)}{4 R^{2}(\Delta r)^{2}}+ & \frac{\left(T_{1}-2\right)^{2}}{(\Delta r)^{4}}+\frac{\nu T_{1}\left(T_{1}-2\right)}{R(\Delta r)^{3}} \\
\frac{\left.\left(1+T_{2}\right) T_{1}+\left(T_{2}-1\right)\left(T_{1}-2\right)\right] \nu}{2 R(\Delta r)^{3}} & +\frac{T_{1}^{2}}{4 R^{2}(\Delta r)^{2}}
\end{array}\right]
$$

where

$$
\begin{aligned}
& T_{1}=\left(\frac{Z_{1} Z_{6}}{Z_{4}}-Z_{3}\right) /\left(Z_{2}-\frac{Z_{1} Z_{5}}{Z_{4}}\right) \\
& T_{2}=\left(Z_{1}-Z_{7}\right) /\left(Z_{2}-\frac{Z_{1} Z_{5}}{Z_{4}}\right)
\end{aligned}
$$

and

$$
\begin{aligned}
& Z_{1}=\frac{1}{\Delta r}+\frac{1}{2 R} \quad Z_{2}=-\frac{3}{\Delta r}-\frac{1}{2 R} \quad Z_{3}=\frac{3}{\Delta r}-\frac{1}{2 R} \\
& Z_{4}=\frac{1}{2 \Delta r} \quad Z_{5}=\frac{\nu}{R}-\frac{1}{2 \Delta r} \quad Z_{6}=-\frac{\nu}{R}-\frac{1}{2 \Delta r} \\
& Z_{7}=-\frac{1}{\Delta r}+\frac{1}{2 R}
\end{aligned}
$$

The system stiffness matrix of the plate $S_{p}$ can be assembled using a procedure equivalent to that in the FEM.

\section{Solution of the problem of vertical oscillations of a flexible plate}

The dynamic equilibrium of the plate for a particular frequency $\omega$ can be expressed as

$$
\left(\mathbf{S}_{\mathbf{p}}-\omega^{2} \mathbf{M}_{\mathrm{p}}\right) \mathbf{w}=\mathbf{P}_{\mathbf{o}}-\mathbf{P}_{\mathbf{s}}
$$

where $\mathbf{M}_{\mathrm{p}}$ is the mass matrix of the plate

$$
\begin{aligned}
& \mathbf{M}_{\mathrm{p}}=\operatorname{diag}\left(A_{j} h_{j} \rho\right)=\rho \mathbf{A H} \\
& A_{j}=2 \pi r_{j} \Delta r \quad \text { for } j \not \equiv 0, \\
& A_{j}=(\Delta r)^{2} \pi / 4 \quad \text { for } j=0
\end{aligned}
$$

and $\rho$ the mass density of the plate. $\mathbf{P}_{\mathrm{o}}$ and $\mathbf{P}_{\mathrm{s}}$ are vectors of equivalent concentrated forces for external

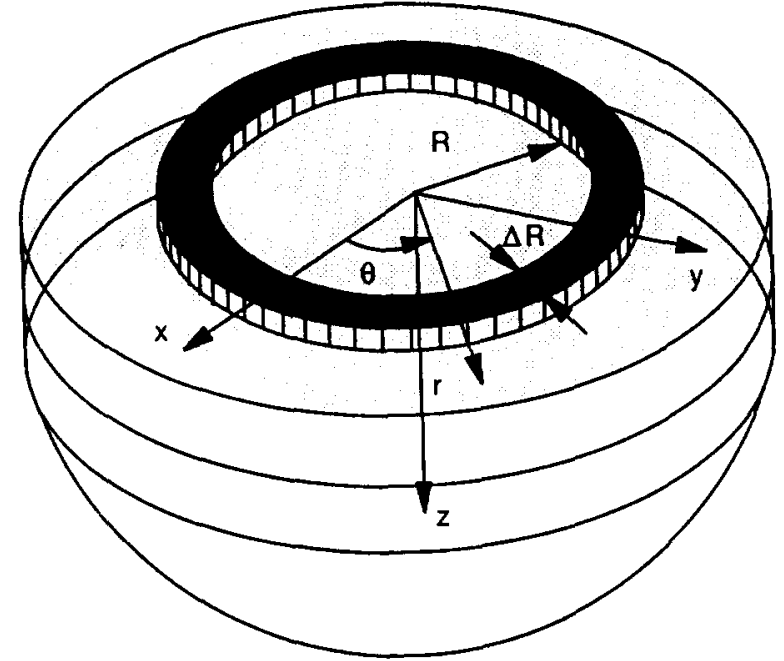

Fig. 5. A schematic of a circular ring at the surface of a layered half-space.

loading and soil reaction, respectively, with components

$$
P_{j}=p_{j} A_{j}, \quad P_{s j}=p_{s j} A_{j}
$$

Vector $\mathbf{P}_{\mathrm{s}}$ can be expressed in terms of surface displacements from the solution of the problem of vertical oscillations of a circular ring at the surface of a layered system, as shown in Fig. 5. This solution can be
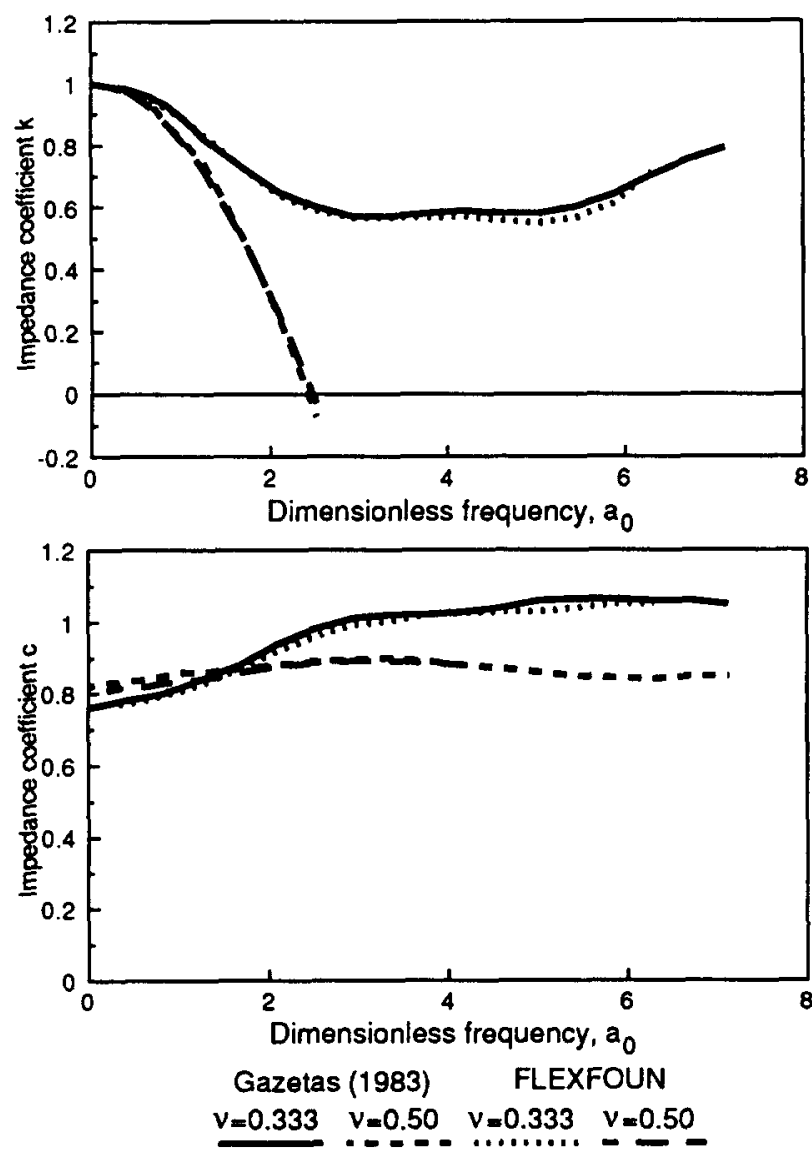

Fig. 6. Impedance coefficients $k$ and $c$ for a rigid disk at the surface of a half-space. 

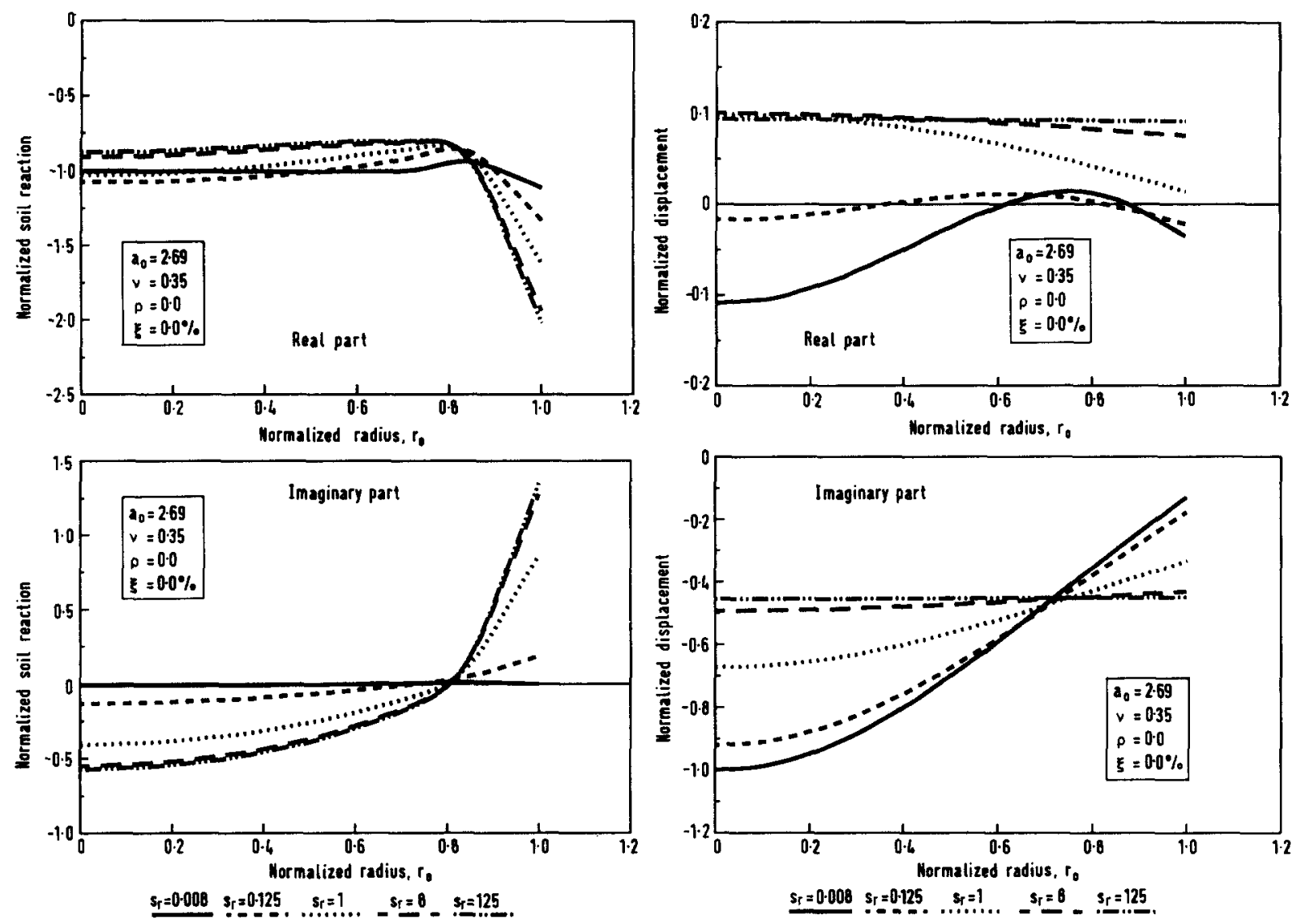

(a)

(b)

Fig. 7. Influence of the stiffness ratio on variation of soil reaction (a) and displacement (b) distributions for a plate on a half-space and $a_{\mathrm{o}}=2 \cdot 69$.

easily derived from the solution of vertical oscillations of a layered elastic half-space due to a circular uniform loading at the surface, described by eqn (5).

In this case the solution can be represented by a superposition of solutions for a uniform vertical circular loading $p_{\mathrm{o}}$ of radius $R+\Delta R$ and loading $-p_{\mathrm{o}}$ of radius $R$. From eqn (3) the surface vertical displacement $w_{\mathrm{s} 0}(r)$ is then equal to

$$
\begin{aligned}
w_{\mathrm{so}}(r)= & p_{\mathrm{o}} \int_{k=0}^{\infty}\left[J_{1}(k(R+\Delta R))(R+\Delta R)\right. \\
& \left.-J_{1}(k R) R\right] k J_{\mathrm{o}}(k r) w_{\mathrm{o}}^{1}(k) \mathrm{d} k
\end{aligned}
$$

Displacement $w_{\text {so }}$ due to the entire set of rings $j=0, \ldots, N$ can be further expressed by the sum

$$
\begin{aligned}
w_{\mathrm{so}}(r)= & \sum_{j=1}^{N}\left(\frac { P _ { 0 j } } { \Delta R _ { j } } \int _ { k = 0 } ^ { \infty } \left[J_{1}\left(k\left(R_{j}+\Delta R_{j}\right)\right)\left(R_{j}+\Delta R_{j}\right)\right.\right. \\
& \left.\left.-J_{1}\left(k R_{j}\right) R_{j}\right] k J_{\mathrm{o}}(k r) w_{\mathrm{o}}^{1}(k) \mathrm{d} k\right)
\end{aligned}
$$

This can be written in the matrix form

$$
\mathbf{L} \mathbf{P}_{\mathrm{s}}=\mathbf{w}
$$

where

$$
\begin{aligned}
L_{i j}= & \frac{1}{\Delta R_{j}} \int_{k=0}^{\infty}\left[J_{1}\left(k\left(R_{j}+\Delta R_{j}\right)\right)\left(R_{j}+\Delta R_{j}\right)\right. \\
& \left.-J_{1}\left(k R_{j}\right) R_{j}\right] k J_{\mathrm{o}}\left(k r_{i}\right) w_{\mathrm{o}}^{1}(k) \mathrm{d} k
\end{aligned}
$$

represents an influence factor for ring $i$ due to ring loading $j$. Radius $r_{i}$ represents the middle of ring $i$ and is equal

$$
r_{i}=R_{i}+\Delta R_{i} / 2
$$

Equation (10) can be combined with eqn (16) to give the solution for the soil reaction vector

$$
\left[\left(\mathbf{S}_{\mathrm{p}}-\omega^{2} \mathbf{M}_{\mathrm{p}}\right) \mathbf{L}+\mathbf{I}\right] \mathbf{P}_{\mathrm{s}}=\mathbf{P}_{\mathrm{o}}
$$

Displacement vector $\mathbf{w}$ can be determined from soil reaction vector $\mathbf{P}_{\mathrm{s}}$ by using eqn (15).

The procedure described has been implemented in program FLEXFOUN, which evaluates displacements below the circular plate for an arbitrary distributed axisymmetric vertical loading and displacements at any point outside the plate which lies at a layer interface. It also evaluates the soil reaction distribution and the impedance of the plate. The impedance is evaluated 
based on the average displacement over the area of the plate, otherwise there would be variable results depending on the selection of the reference point. The validity of the procedure and operation of the program were verified by comparing the impedance function results for a rigid circular footing on an elastic halfspace with the analytical solution. Figure 6 shows a comparison of impedance coefficients $k$ and $c$ taken from Gazetas ${ }^{2}$ with those obtained by the program. A plate with ten rings was used in the analysis. Gazetas used the definition of the impedance of the form

$$
K=K_{\mathrm{s}}\left(k+\mathrm{i} a_{\mathrm{o}} c\right)(1+2 \mathrm{i} \xi)
$$

where $K_{\mathrm{s}}$ is the static stiffness and $a_{\mathrm{o}}$ the dimensionless frequency defined by

$$
a_{\mathrm{o}}=\frac{\omega R_{\mathrm{o}}}{V_{\mathrm{s}}}
$$

The static stiffness of the disc is equal to

$$
K_{\mathrm{s}}=\frac{4 G R_{\mathrm{o}}}{1-\nu}
$$

The static stiffness obtained from the program differs less than $2 \%$ from the stiffness defined by eqn (22).
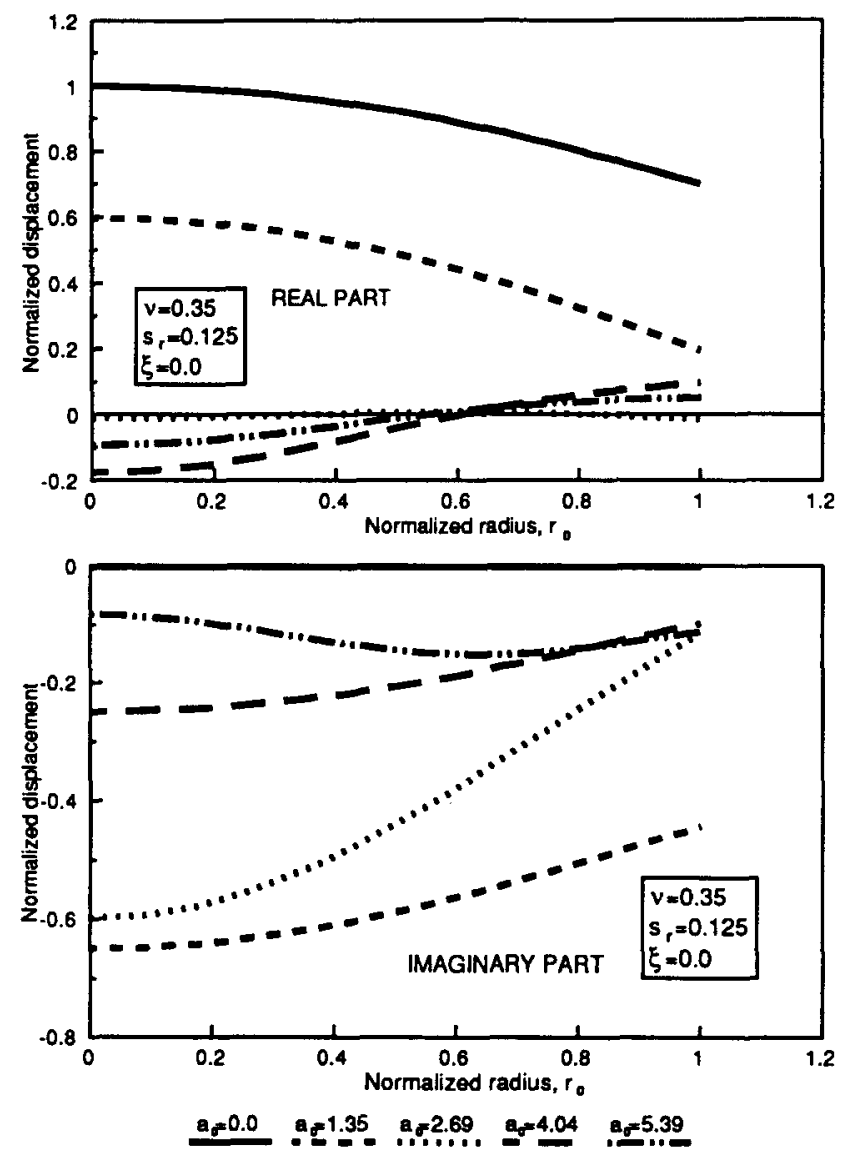

Fig. 8. Plate sections and load distributions used in the analysis.

\section{NUMERICAL EXAMPLES}

The examples included illustrate the influence of the stiffness of the plate relative to the stiffness of a soil medium, the geometry and mass of the plate, and the loading distribution on the response of the plate. Even though the program can analyze soil systems with an arbitrary number of layers, the examples herein are limited to cases of a flexible plate at the surface of a half-space, and a layer over a half-space system.

Figures 7(a) and 7(b) illustrate the influence of the stiffness ratio on the soil reaction and displacement distributions below the plate at the surface of a halfspace for $a_{\mathrm{o}}=2.69$. The stiffness ratio, $s_{\mathrm{r}}$, represents the ratio of the stiffness of the plate and the stiffness of the soil medium subjected to the surface vertical loading, and is defined as $s_{\mathrm{r}}=E_{\mathrm{p}} h^{3} /\left(\rho_{1} V_{\mathrm{s} 1}^{2} R_{\mathrm{o}}^{3}\right) . V_{\mathrm{s} 1}$ and $\rho_{1}$ are the shear wave velocity and the mass density of the surface layer, in this case the half-space. The other variables used in the presentation of the results are: normalized radius $r_{\mathrm{o}}=r / R_{\mathrm{o}}$, normalized soil reaction $p_{\mathrm{o}} / p_{\mathrm{s}}$, and normalized displacement $w /\left|w_{\max }\right|$. The Poisson's ratio of the plate $\nu_{\mathrm{p}}$ is kept constant with a value 0.25 and the plate is divided into ten rings in this and all other cases. The plate is massless, which is defined through the mass density ratio $\bar{\rho}=\rho h /\left(\rho_{1} R_{\mathrm{o}}\right)$ equal to zero, of the uniform thickness and with uniformly distributed loading. Significant decrease of the soil reaction concentration in the vicinity of the edge and strong variations of the displacements in the radial direction can be observed for $s_{\mathrm{r}}$ equal to or less than $0 \cdot 125$. On the other hand, an almost uniform displacement distribution, typical for the response of rigid plates, can be observed for $s_{\mathrm{r}}$ equal to or greater than 8 . Soil

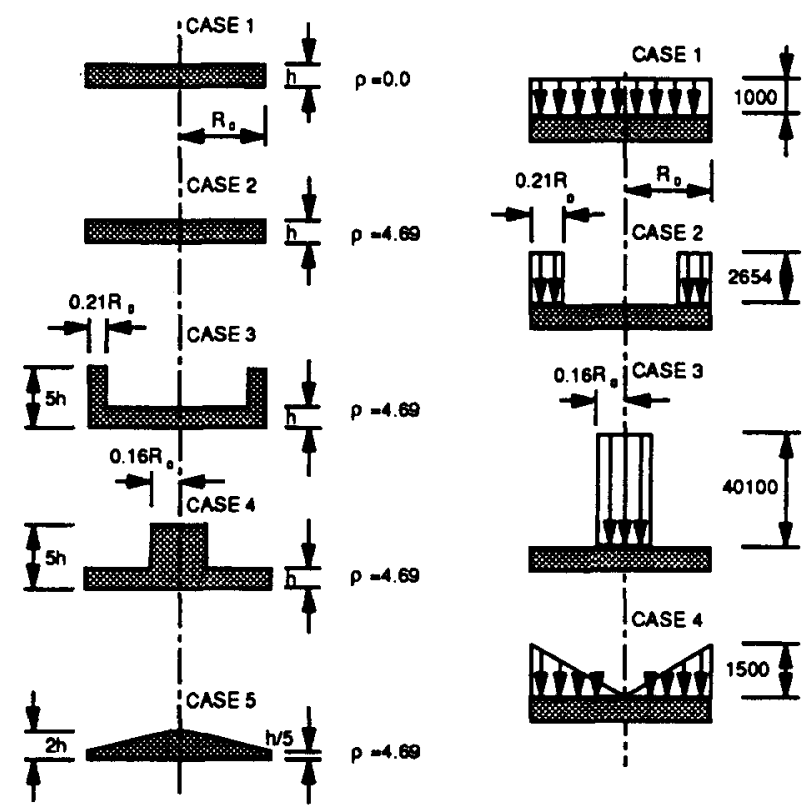

Fig. 9. Variation of displacements with frequency for a plate on a half-space and $s_{\mathrm{r}}=0 \cdot 125$. 

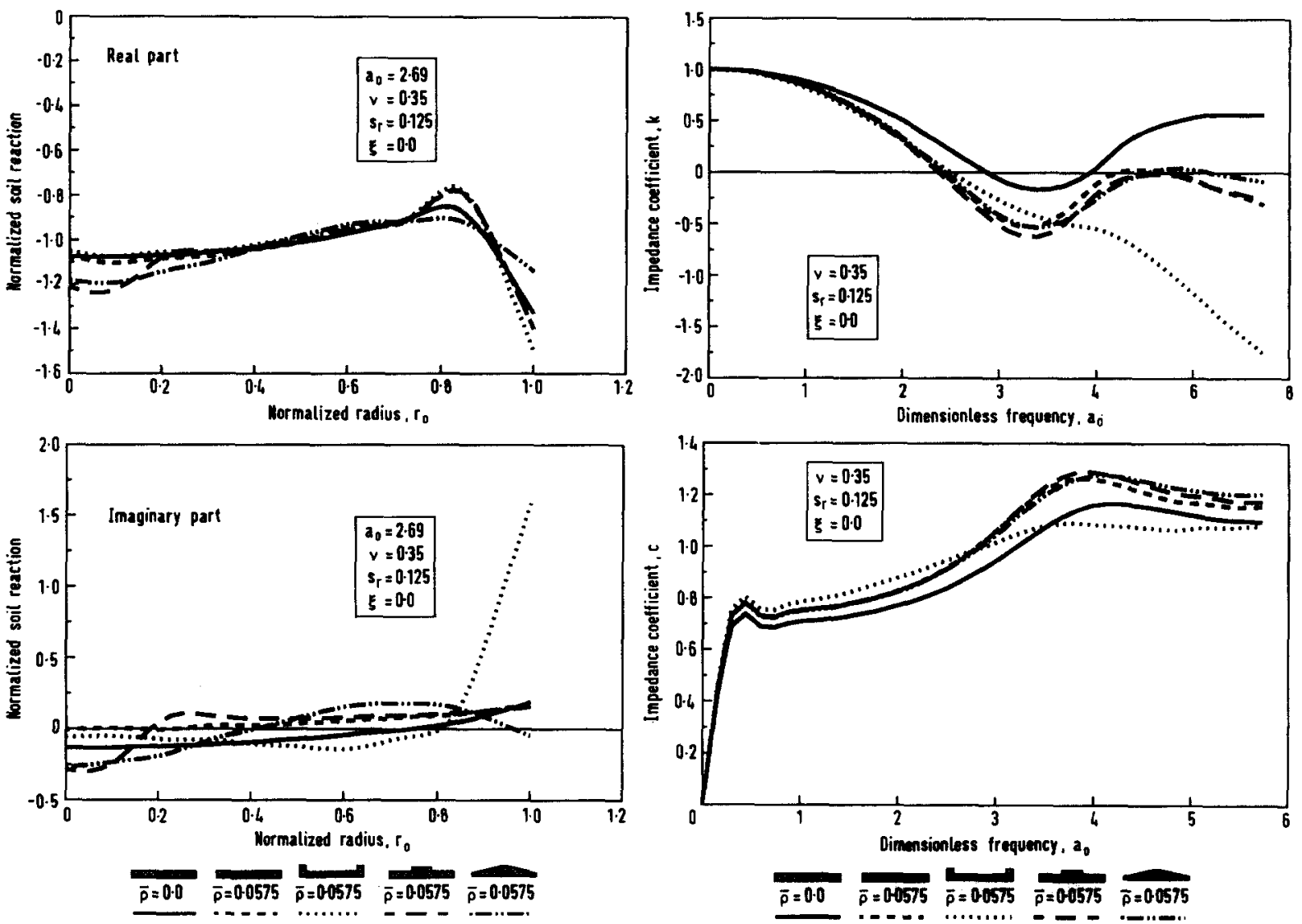

(a)

(b)

Fig. 10. Influence of the mass and stiffness distribution on variation of soil reaction distribution for $a_{0}=2.69$ (a), and impedance coefficients $k$ and $c(\mathrm{~b})$.
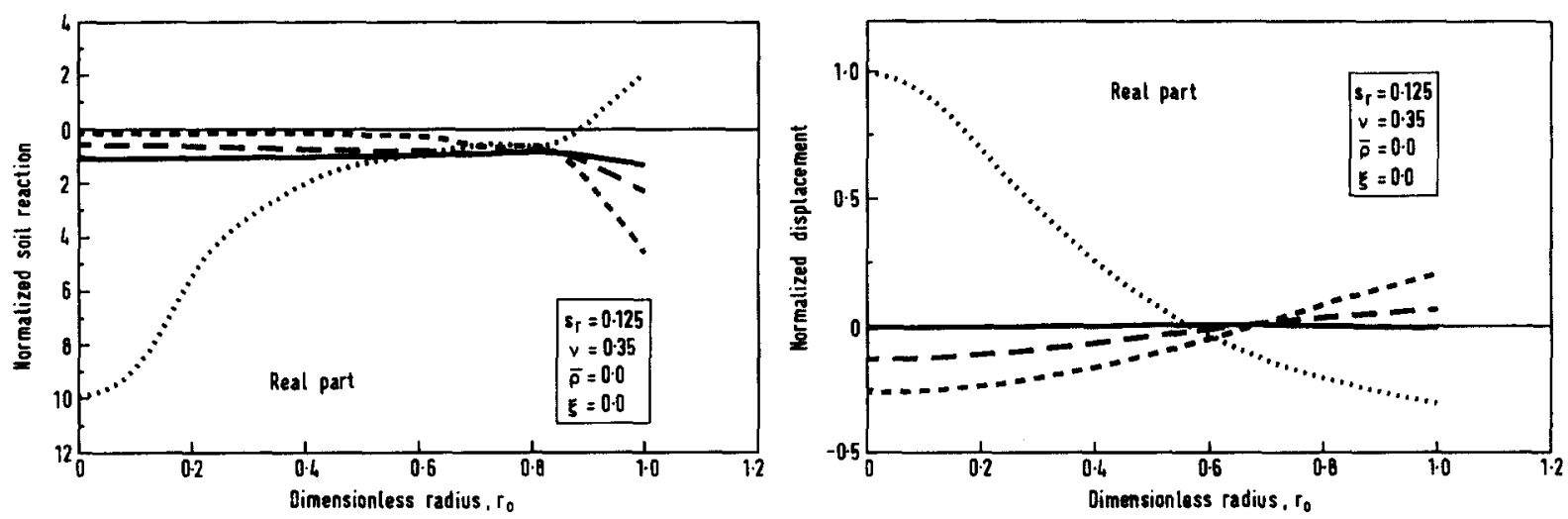

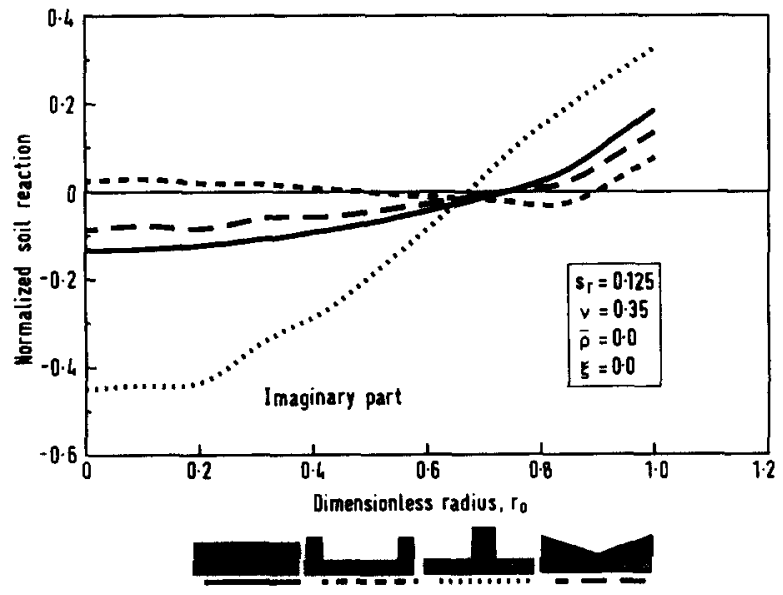

(a)

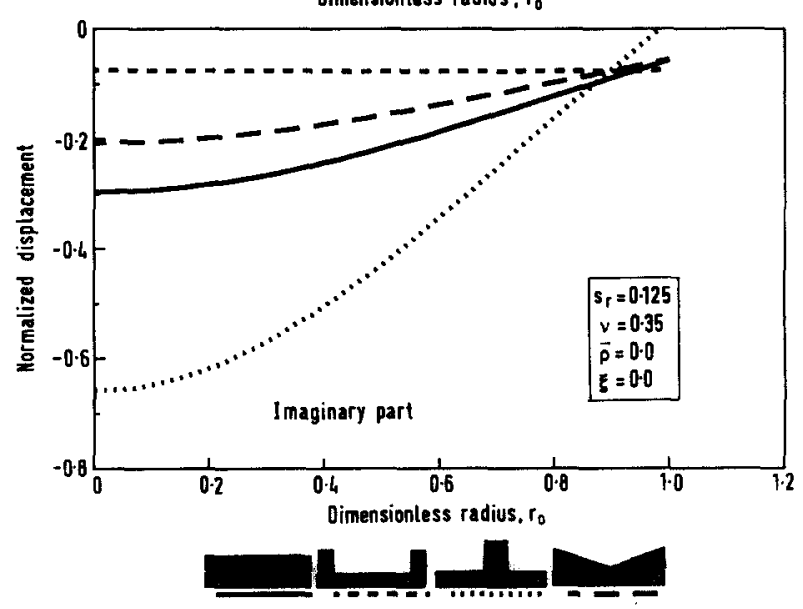

(b)

Fig. 11. Influence of the loading distribution on soil reaction (a) and displacement (b) distributions for a plate on a half-space and $a_{\mathrm{o}}=2 \cdot 69$. 

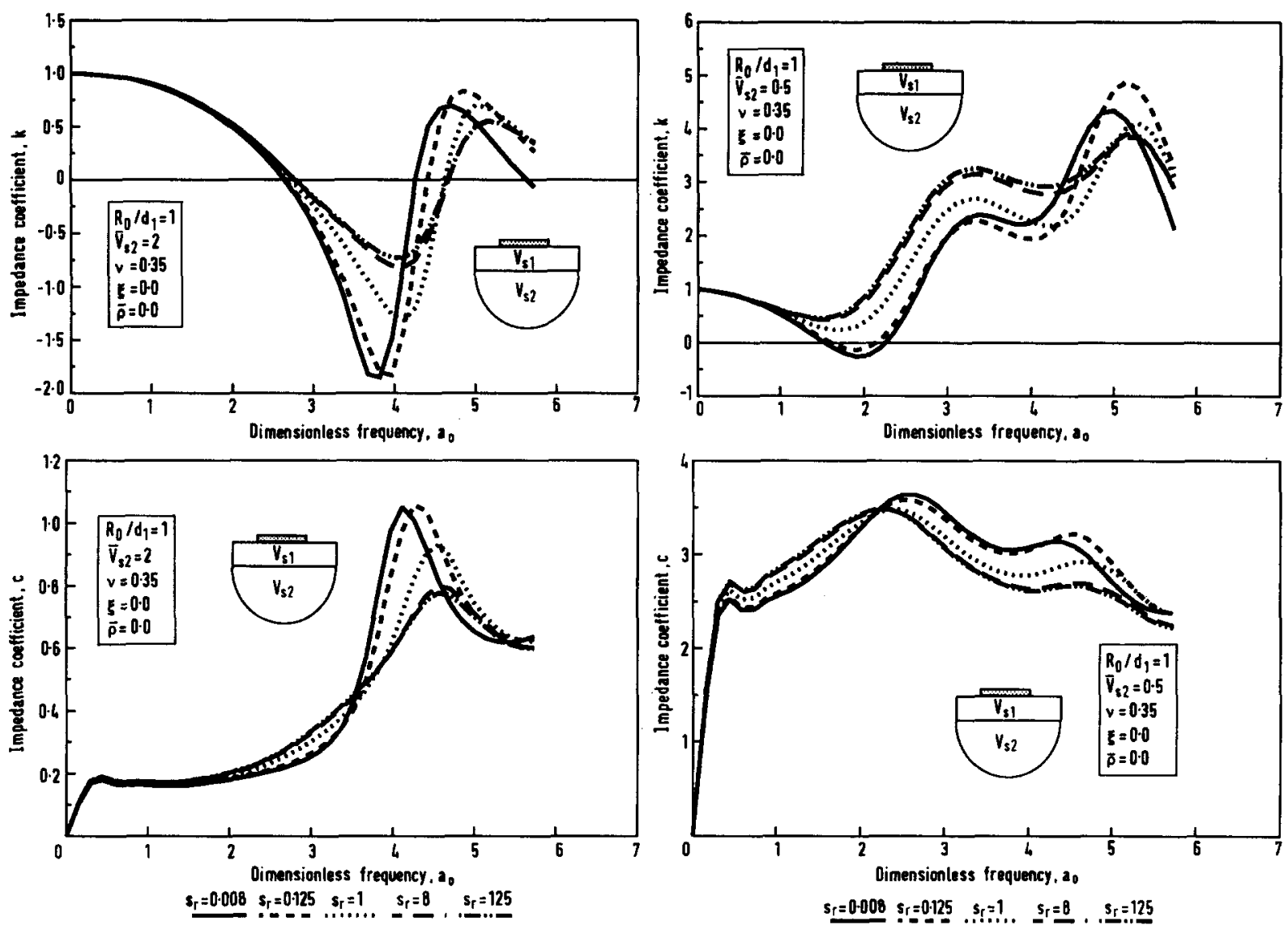

(a)

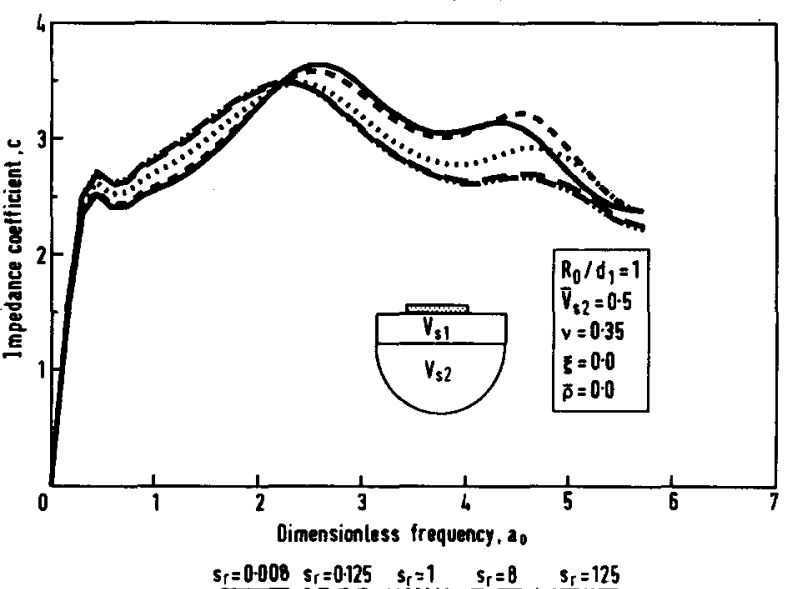

(b)

Fig. 12. Influence of the stiffness ratio on variation of impedance coefficients $k$ and $c$ for a plate on a layer underlain by a stiffer (a) and a softer half-space (b).

reaction and displacement distributions vary with frequency, especially for low values of the stiffness ratios. This is illustrated for displacements in Fig. 8.

Figure 9 shows cases that illustrate the influence of mass and stiffness distribution of the plate and load distribution in the radial direction on vertical oscillations of the plate. The influence of the mass and stiffness distribution on the soil reaction distribution and impedance coefficients is illustrated in Figs 10(a) and $10(\mathrm{~b})$, respectively. The stiffness ratio of all the plates is 0.0125 and is based on the marked thickness $h$ in Fig. 9. All the plates, except for the massless plate of case 1 , have a mass density ratio equal to 0.0575 . The soil reaction distribution of all the cases is similar to the one for the massless plate, except for the plate with the rigid edge which has a distribution similar to the rigid plate (Fig. 7(a)). Similar behavior can be described for the impedance. Cases 2, 4 and 5 have very close impedances, while cases 1 and 3 differ from those due to different plate mass and due to the rigid edge, respectively.

Influence of the load distribution on the soil reaction and displacement distributions is illustrated in Figs 11(a) and 11(b), respectively, for $s_{\mathrm{r}}=0.125$. The condition for the loading cases is that the total loading in all four cases is equal. Variations of both distributions are pronounced in cases with concentrated loadings at the center and the edge. For a stiffness ratio equal to or greater than 8 , not shown here, the variations are small.

Finally, the influence of the stiffness ratio on the impedance of a flexible plate at the surface of a layer over a half-space system is demonstrated in Fig. 12. The system in Fig. 12(a) represents the case when the layer is underlain by a stiffer half-space, while the system in Fig. 12(b) represents the case when the layer is underlain by a softer half-space. The dimensionless frequency in this case is defined as $a_{\mathrm{o}}=\omega R_{\mathrm{o}} / V_{\mathrm{s} 1}$, where $\bar{V}_{\mathrm{s} 2}$ represents the ratio of velocities $V_{\mathrm{s} 2}$ and $V_{\mathrm{s} 1}$. Variations of the impedance coefficients in the first cases become more pronounced for $a_{\mathrm{o}}$ greater than about 3 , which corresponds, approximately, to the first natural frequency of vertical oscillations of the layer over a rigid bedrock. In the second case the variations become more pronounced at a much lower dimensionless frequency.

\section{SUMMARY AND CONCLUSIONS}

The solution to the problem of vertical oscillations of a flexible circular foundation at the surface of an elastic 
layered half-space has been presented. The solution is based on the stiffness-matrix approach for the definition of the influence coefficient matrix for the soil system, and the finite difference energy method for the definition of the stiffness matrix of the plate. Numerical examples presented indicate that the response of a flexible plate depends on several parameters, and in most cases significantly differs from the response of a rigid plate. Some of the parameters presented include the stiffness ratio, mass and stiffness distribution of the plate, the loading distribution, and the soil stratification.

\section{REFERENCES}

1. Richart, F.E. Jr, Hall, J.R. Jr, \& Woods, R.D. Vibrations of Soils and Foundations, Prentice-Hall, Englewood Cliffs, NJ, 1970, 414 pp.

2. Gazetas, G. Analysis of machine foundation vibrations: state of the art, Soil Dynamics and Earthquake Engineering, $1983,2,2-42$.

3. Luco, J.E. Impedance functions for a rigid foundation on a layered medium, Nuclear Engineering and Design, 1974, 31, 204-17.

4. Luco, J.E. Vibrations of a rigid disc on a layered viscoelastic medium, Nuclear Engineering and Design, 1976, 36, 312-26.

5. Kausel, E. An explicit solution for the green functions for dynamic loads in layered media, MIT Research Report R 81-13, Order No. 699, Department of Civil Engineering, MIT, Cambridge, MA, 1981.

6. Tassoulas, J.L. Elements for the numerical analysis of wave motion in layered media, MIT Research Report $\mathbf{R}$ 81-2, Order No. 689, Department of Civil Engineering, MIT, Cambridge, MA, 1981.

7. Wolf, J.P. \& Darbre, G.R. Dynamic-stiffness matrix of surface foundation on layered half-space based on stiffness-matrix approach, in Summary Report, IAEA Specialists Meeting on Gas-Cooled Reactor Seismic Design Problems and Solutions, IAEA IWGGCR/6 UC-77, General Atomic Company, San Diego, CA, 1983, pp. 183-206.

8. Lin, Y.L. Dynamic response of circular plates resting on viscoelastic half space, Journal of Applied Mechanics, ASME, 1978, 40, 379-84.
9. Iguchi, M. \& Luco, J.E. Dynamic response of flexible rectangular foundations on an elastic half-space, Earthquake Engineering and Structural Dynamics, 1981, 9, 239-49.

10. Iguchi, M. \& Luco, J.E. Vibration of flexible plate on viscoelastic medium, Journal of Engineering Mechanics Division, ASCE, 1982, 108(10), 1103-20.

11. Riggs, H.R. \& Waas, G. Influence of foundation flexibility on soil-structure interaction, Earthquake Engineering and Structural Dynamics, 1985, 13, 597-615.

12. Karabalis, D.L. \& Beskos, D.E. Dynamic response of 3-D flexible foundations by time domain BEM and FEM, Soil Dynamics and Earthquake Engineering, 1985, 4(2), 91-101.

13. Gaitanaros, A.P. \& Karabalis, D.L. Dynamic analysis of 3-D flexible embedded foundations by a frequency domain BEM-FEM, Earthquake Engineering and Structural Dynamics, 1988, 16, 653-74.

14. Swaddiwudhipong, S., Chow, Y.K., Tan, S.C. \& Phoon, K.F. Dynamic response of surface foundations on layered media, Earthquake Engineering and Structural Dynamics, 1991, 20, 1065-81.

15. Lysmer, J. Vertical motion of rigid footings, US Army Engineer Waterways Experiment Station, Report No. 3-115, Vicksburg, Mississippi, 137 pp, 1965.

16. Waas, G. Dinamisch belastete Fundamente auf geschichtetem Baugrund, VDI Berichte, 1980, 381, 185-9.

17. Kausel, E. \& Roesset, J.M. Stiffness matrices for layered soils, Bulletin of Seismological Society of America, 1981, 71(6), 1743-61.

18. Thomson, W.T. Transmission of elastic waves through a stratified soil medium, Journal of Applied Physics, 1950, 21, 89-93.

19. Haskell, N.A. The dispersion of surface waves in multilayered media, Bulletin of Seismological Society of America, 1953, 43(1), 17-43.

20. Wolf, J.P. \& Obernhuber, P. Free-field response from inclined SV- and P-waves and Rayleigh-waves, Earthquake Engineering and Structural Dynamics, 1982, 10, 847-69.

21. Wolf, J.P. Dynamic Soil-Structure Interaction, PrenticeHall, Englewood Cliffs, NJ, 1985, 466 pp.

22. Gucunski, N. \& Woods, R.D. Numerical simulation of the SASW test, Soil Dynamics and Earthquake Engineering, 1992, 11(4), 213-27.

23. Bushnell, D. Computerized analysis of shells-governing equations, Technical Report AFWAL-81-3048, Applied Mechanics Laboratory, Lockheed Palo Alto Research Laboratory, Palo Alto, CA, 1981. 